\title{
Fever due to infection or muscle heat?
}

\begin{abstract}
Paroxysmal sympathetic hyperactivity is a frequent condition in patients that suffered a TBI, but also other kinds of brain damage, characterized by a myriad of signs and symptom such as high temperature, tachycardia, tachypnea, hypertension, profuse sweating and spastic extension of legs and arms. The presence of high temperature may lead to suspect about any infective process whose analysis can put at risk the life of the patient, while in fact these symptom appear as a consequence of an increased release of catecholamines occurring because of the disruption of central pathways. Muscle spasticity produces a great heat that contributes to the central origin of hyperthermia. Here we describe two cases that presented PSH after different brain injuries. Both were in coma. One of them after a severe TBI and the other one because of a very important brain infection. PSH in the first case was due to a renal colic and PSH was resolved with propofol. The second case developed PSH suddenly before a session of physiotherapy. The rapid detection of the problem led us to use propranolol and in 40minutes the patient was in normal conditions. We conclude that the knowledge of PSH and its rapid treatment should be mandatory in hospitals and centers devoted to rehabilitate neurological patients.
\end{abstract}

Keywords: traumatic brain injury, paroxysmal sympathetic hyperactivity, hyperthermia, tachycardia, tachypnea, muscle heat, catecholamines, growth hormone
Volume 3 Issue 6 - 2017

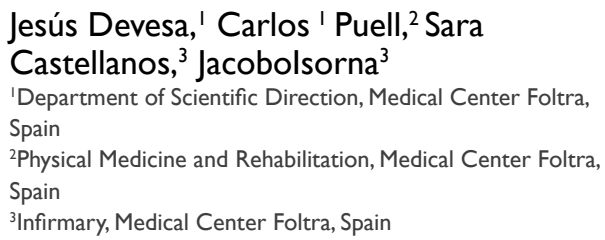

Correspondence: Jesús Devesa, Scientific Direction, Medical Center Foltra,Travesía de Montouto 24, I5886 Teo, Spain, Tel +34981802928, Email jesus.devesa@usc.es

Received: June 13, 2017 | Published: June 29, 2017
Abbreviations: TBI, traumatic brain injury; DAI, diffuse axonal injury; GH, growth hormone; PSH, paroxysmal sympathetic hyperactivity; ICU, intensive care unit; MRI, nuclear magnetic resonance; GHRH, growth hormone releasing hormone

\section{Introduction}

It is relatively frequent that patients who suffered a traumatic brain injury (TBI), particularly those in which the damage resulted in diffuse axonal injury (DAI), suddenly present a strong sympathetic discharge which may endanger their life. Usually this situation affects up to $1 / 3$ of patients with severe TBI. ${ }^{1}$ Theseparoxistic autonomic activities have been described to occurdays or weeks after $\mathrm{TBI},{ }^{2}$ however they can also appear for the first time even 1-2years after the brain injury, as we will describe in this report. Symptoms of paroxistic autonomic activity can be present duringmonths oryears, ${ }^{3}$ there is even study demonstrating that this process can have a mean duration of 5years after the injury. ${ }^{4}$ Even more, although they are mainly related to TBI, they can occur in other kinds of patients with brain damage without DAI or in patients with spinal cord injury.

Symptoms of what first was termed hypothalamic or sympathetic storm, and now is known as Paroxysmal sympathetic hyperactivity (PSH), according to the proposal of a consensus workgroup organized by the International Brain Injury Association for establishing the nomenclature and diagnostic criteria of thisdays autonomic problem, ${ }^{5}$ mainly consists of:" a "syndrome, recognised in a subgroup of survivors of severe acquired brain injury, of simultaneous, paroxysmal transient increases in sympathetic [elevated heart rate, blood pressure, respiratory rate, temperature, sweating] and motor [posturing] activity" ${ }^{6}$ However, while these symptoms are standard in its presentation, its presentation may occur in other kind of patients, as stated above. For instance they can be shown in patients with cranial hypertension produced by hydrocephalus, or a brain tumor, or simply by a strong pain whose origin cannot be described by the patient. In addition, the severity of the symptoms is very variable, and mydriasis seems to be a constant, due to the increased release of catecholamines producing the excitation of the radial fibres of the iris.

Still there are not clear reasons as to why PSH occurs; this is because there are not clear explanations for the variability of the occurrence observed in some patients. These may increase the morbidity of the patients when they have been discharged from the Intensive Care Unit (ICU), or the hospital and they are at home or receiving rehabilitation. An early diagnosis of the situation is critical to take appropriate measures to correct this emergency which can compromise the life of the patient.

Here we will describe two different cases in which PSH occurred, as well as how emergency measures adopted saved the life of the patients that were seriously compromised in both cases.

\section{Case presentation}

\section{Case I}

Male, 52years old. Sixmonths before being treated in our Center he suffered a traffic accident resulting in severe TBI, polytrauma and coma Glasgow 4. He was referred to a level 3 hospital. Nuclear magnetic resonance revealed the existence of DAI and a subluxation of the second cervical vertebra deforming the normal anatomy of the brainstem. The patient remained in the ICU of that hospital during 4 months without any change in his condition, excepting for a number of pneumonia typical of ICUs (Pseudomonaaeroginosus, staphylococcus, etc). After this time he was discharged, without changes in Glasgow scale, for doing rehabilitation at home.

Sevenmonths after the accident the patient was admitted to Medical Center Foltra and after clinical, radiological examination and blood analysis (hematimetry, biochemistry, tumoral markers and pituitary hormones) the patient was scheduled for medical treatment and rehabilitation. 
Medical treatment consisted of:

a. GH (Nutropín, Ipsen, Spain), 0.8mg/day, 5days/week, during threemonths, followed by one month resting and again the same protocol. Treatment with GH lasted 1year;

b. Melatonin (masterful preparation), at a daily dose of $50 \mathrm{mg}$, via gastric tube, at $9.30 \mathrm{pm}$;

c. Baclofen, (Lioresal, Novartis), at a daily dose of $20 \mathrm{mg}$ every 8hours). Medical treatments were conducted after obtaining signed informed consent of the legal representative of the patient and according to the protocols of Medical Center Foltra in compliance with national legislation and the Code of Ethics of the World Medical Association (Declaration of Helsinki).

Rehabilitation consisted of:

i. Physiotherapy, two sessions/day, 5days/week;

ii. Speech therapy, 1session/day, 5days/week;

iii. Occupational therapy, 1 session/day, 5 days/week;

iv. Psychomotor stimulation, 1session/day, 5days/week;

v. Visual stimulation with a tachistoscope, 1session/day, 5days/ week.

All sessions lasted 1hour with the exception of visual stimulation (15minutes). In addition, the patient was placed daily in a vertical plane for 30minutes in order to control blood pressure and prevent the appearance of orthostatic hypotension.

At admission the patient remained in coma, Glasgow 4. He presented a high spasticity, score 4 in the Modified Ashworth scale, with legs in extension, equine varus feet, and arms in almost irreducible flexion. He lacked cephalic and trunk control. He had to be fed by a gastric tube and he was fully unable to respond to any order.

8 months later the patient had acquired cephalic and trunk control, he was able to sit in a chair, he responded to questions by closing his eyes once (yes) or twice (no), he began to swallow water with thickener and he responded to phone calls from friends talking to him in different languages although his words lacked intelligibility. Glasgow score at this time increased until a value of 10 .

Despite these improvements, one day we were called urgently by his caregivers (it was a weekend and the patient was at home), because suddenly fever appeared reaching $38.5^{\circ} \mathrm{C}$ despite the fact that they had been administered antipyretics (paracetamol) to him. Ten minutes later temperature had been increased to $39.2^{\circ} \mathrm{C}$. The patient was tachycardic, he presented a very high spasticity, profuse sweating and lack of awareness. We called to emergencies while covering him with ice cubes. Fifteen minutes later the patient entered the critical patients unit of a level 3 hospital. At this time his temperature was $41^{\circ} \mathrm{C}$. He was given a number of antipyretics but the temperature continued to increase until reaching $42^{\circ} \mathrm{C}$. There was no time for waiting for the results of the blood analysis for detecting any infection. Moreover, no signs of it existed in the explorations carried out. Then, we thought that perhaps it was not fever but muscle heat due to the high spasticity; in turn, this could be produced by a strong pain of unknown origin. Hence we asked the chief of critical patients unit to give him a strong pain relieving or a muscle relaxant drug. He accepted and administered propofol intravenously to the patient. Fifteen minutes later temperature was $36.2^{\circ} \mathrm{C}$, a suggestive data that the patient did not have fever because of an infection, but increased temperature produced by the spasticity and the consequent production of muscle heat.
Clinical, radiological and laboratory studies performed in the following hours revealed that the patient had suffered a renal colic. Since he could not express what was happening due to the lack of intelligible communication, it seems to be clear that in this case it was the strong pain, in his condition, that was the cause responsible for the strongly increased temperature.

Unfortunately, the prolonged high temperature led to maintain during a time led him back in the improvements he was reaching, because of a further brain damage that never could be recovered.

\section{Case 2}

Male, 31years old. According to the reports provided by the family, twoyears before his admission in Medical Center Foltra he suffered a frontal sinusitis that was treated with amoxicillin $875 \mathrm{mg} /$ clavulanic $125 \mathrm{mg}$. Tendays after commencing this treatment the infection progressed and the patient presented retro-orbicular pain, decreased consciousness, left palpebral ptosis and aphasia. A brain CT-SCAN detected a left frontotemporal subdural collection with mass effect. Antibiotics were changed but the general situation of the patient continued to worsen and a new brain CT-SCAN detected a left extra-axial frontotemporal subdural collection with meningeal enhancement, mass effect and collapse of the left lateral ventricle. The patient underwent surgery for drainage of the left frontal sinus, anterior etmoidal sinus and left maxillary sinus. Given the existence of cranial hypertension, a craniotomy was performed and pus was collected and analyzed. The analysis indicated the existence of Staphylococcus aureus, Eikenella corrodens and Streptococcus anginosus.

A MRI performed 6months later found supratentorial ischemic injuries in both brain hemispheres and in the brainstem, with necrosis in most of these injuries. During the time that the patient was in this hospital he suffered one episode of pulmonary thromboembolism and a deep venous thrombosis in the right leg. Interestingly, an episode of PSH occurred during his stay in this hospital, as the family told to us.

The patient received daily rehabilitation (physiotherapy and psychostimulation) without any change in his coma Glasgow 4. Therefore, the patient was discharged from that hospital and went to rehabilitation to another center for 1year, after it he was discharged without changes.

At admission in the Medical Center Foltra, 18months after his sinusitis, the patient remained in Glasgow 4 and his score in the Modified Ashworth scale was 4; he was fed by gastric tube, he did not respond to any order, legs and arms were in extension, pupils practically lacked any reactivity, he did not follow the light. After obtaining signed informed consent of the legal representative of the patient and according to the protocols of Medical Center Foltra in compliance with national legislation and the Code of Ethics of the World Medical Association (Declaration of Helsinki) we scheduled a medical treatment consisting in: $\mathrm{GH} 1 \mathrm{mg}$ /day (similar to the protocol followed in Case 1), Melatonin 50mg/day, and rehabilitation: Physiotherapy, Speech therapy, Occupational therapy, Psychomotor stimulation, Visual stimulation, Auditive stimulation with headphones and acoustic frequencies previously selected, Pool therapy (assisted by a therapist) and a special session (1/week) of multisensory stimulation carried out together with another patients in coma. Blood analysis and control of the evolution were scheduled to be performed every 3 months.

After 1month under this treatment the patient slightly improved in his condition, being the Glasgow score 7. However, one morning just before commencing his session of physiotherapy, the therapist brought him to the infirmary of the Center, because she observed a 
profuse sweating, tachypnea and stronger spasticity. The patient was immediately monitored electrocardiographically and medical doctors were called. No signs of lung infection or any other cause responsible for the situation existed, but there was tachycardia (184bpm), arrhythmias, mydriasis, tachypnea (36/min), hypertension (17/12), high temperature $\left(39.7^{\circ} \mathrm{C}\right)$, and irreducible spasticity in extension (arms and legs), all of them clear signs of PSH. We immediately gave him propranolol (Sumial 20mg) and called to Emergencies for his transfer to a level 3 hospital. When he arrived to the hospital and while he was waiting for attention (approximately 40minutes since PSH commenced), his situation was totally normal. Cardiac frequency was $84 \mathrm{bpm}$, no tachypnea existed, temperature was $36.2^{\circ} \mathrm{C}$, sweating had disappeared, and the patient looked more relaxed. Medical doctors in Emergencies initially prescribed antibiotics, but then they changed to an analgesic if it was needed. Blood analysis demonstrated the lack of any infection. Therefore, the patient was discharged and we decided to continue with propranolol $20 \mathrm{mg}$ every 7 hours during 3 days.

As in Case 1, it is clear that in this case high temperature was produced by muscle heatand/or lack of the hypothalamic control, and it is clear too that given the situation of the patient (high tachycardia and hypertension), the rapid decision to prescribe the beta 2-adrenergic receptor blocker avoided a fatal outcome. Currently the patient went back to his daily therapies without any problem.

\section{Discussion}

The existence of PSH has been known for over sixty years. In 1988 Boeve et al. ${ }^{6}$ described a case of a patient that presented paroxysmal sympathetic storms after having suffered a very important brain injury. These authors found that these sympathetic storms presented characteristics similar to those described in "diencephalic seizures". However, electroencephalogram showed that no epileptiform activity existed and antiepileptic drugs failed to control the symptoms, while morphine effectively controlled them. From their data they concluded that sudden changes in intraventricular pressure or activation of reflexes triggered from muscle mechanoreceptors or chemoreceptors occurring during episodes of spasticity had to be the most feasible cause for this Paroxysmal sympathetic storms. In the same year, it was described "The autonomic dysfunction syndrome" in nine patients relating it to severe brain injury and acute hydrocephalus. Three of these patients were treated with morphine which stopped the episodes; bromocriptine was given to other three patients resulting in beneficial short-term and long-term effects. Dantrolene (a muscle relaxant drug) was given to one of these patients, decreasing the severity of the extensor posturing without affecting the other components of the autonomic dysfunction syndrome; this seems to exclude muscle heat as a reason for hyperthermia. From these data, authors concluded that the syndrome was produced by a lack of control of the brainstem from the control of higher brain centers. Moreover, they suggested that the dopaminergic and opiate pathways should play a role in the development of the syndrome. Responsiveness to bromocriptine and morphine had been previously reported in what had been termed "diencephalic seizures". ${ }^{8}$

The reasons by which PSH occurs and its morbid effects have been discussed duringyears, not only in adult TBI patients but also in pediatric populations and other pathologies. ${ }^{9-16}$ Recently Meyer $^{3}$ propose that PSH courses in three stages being the first of them asymptomatic because of the strong sedation of the patient and the management of cranial hypertension, the second stage would correspond to the onset of symptom clusters and the third stage would be characterized by dystonia and a decline in posturing. However, what is the reason for the appearance of PSH? Most of the theories proposed suggest that as a consequence of the damage suffered by the brain there is an anatomic disconnection between mesencephalon and the spinal cord/hypothalamus leading to a disruption in relay from these last structures. ${ }^{3}$ Another theory implies the affectation of the Excitatory: Inhibitory ratio model. This affectation would mean that the dysfunction of the diencephalic-brainstem inhibitory center, which physiologically controlsthe processing of afferent stimulus in the spinal cord, would be the reason for PSH. ${ }^{17-19}$ That is, the appearance of an autonomic dysfunction would be related to the brain damage resulting in functional disconnections involving deep and important brain centers; moreover, a significant reduction in dopaminergic activity would worsen the clinical picture, further worsened by the resulting unopposed adrenergic outflow with increased levels of plasma catecholamines. ${ }^{3}$ In summary, this hypothesis of Meyer ${ }^{3}$ would indicate that in PSH there will be an increase in the excitatory stimuli without the needed compensatory increase in inhibitory functions. This would lead to facilitation to develop a highly exaggerated sympathetic efflux triggered by non-nociceptive peripheral stimuli. ${ }^{4-20}$

More recently, a diffusion tensor imaging analysis performed in 102 patients that suffered severe TBI three weeks prior to the study showed that patients with PSH had a severe and diffuse injury to white matter tracts; however, disruption within the central autonomic network seems to play a key role in the pathogenesis and development of $\mathrm{PSH}^{21}$ One of these disrupted pathways seem to be the fibres of the right insula via damage to the posterior limb of the ipsilateral internal capsule. ${ }^{21}$ These authors conclude that disconnection involving the posterior corpus callosum and of the posterior limb of the internal capsule may be responsible for the pathogenesis or appearance of PSH.

In the case of the patients we described here it could be argued that $\mathrm{GH}$ treatment might have been the reason for the expression of PSH. In fact, a possible adverse effect of $\mathrm{GH}$ is the production of mild cranial hypertension. Moreover, GH is mainly regulated by catecholamines which negatively regulated somatostatin release from the hypothalamus and induce GHRH releasethat in turn stimulates pituitary GH secretion in the absence of the negative control exerted by somatostatin. ${ }^{22-26}$ In addition, GH secretion or exogenously administered increases the release of somatostatin which, in turn, leads to enhanced hypothalamic adrenergic secretion. A schematic representation of this concept is shown in Figure 1. Therefore, it would be possible that given that our patients receive GH treatment for increasing brain repair, ${ }^{26-30}$ the hormone might have been the factor responsible for PSH. However, this possibility has to be discarded since in the first case the patient had not been treated with the hormone because of being weekend, and in the second case PSH occurred before the daily administration of GH would have taken place. Moreover, we treated more than 80 patients with severe TBI, 22 in coma, and much other neurological pathology without any problem. Although in our protocols the detection of a possible GH-deficiency occurred after a TBI is not included, because in these patients any provocative test among those commonly used is risky, we know that if no GHdeficiency exists GH administration cooperates with the endogenous hormone in increasing the proliferative response of neural progenitors and their differentiation to neurons after a brain injury. ${ }^{31}$

Since NA is formed from DA, the administration of the dopaminergic agonist bromocriptine would reduce DA synthesis, therefore decreasing noradrenaline and adrenaline levels. This might explain the responsiveness to bromocriptine observed in one study about PSH. ${ }^{8}$ 
In summary, when a possible PSH is detected medical action must be swift. In our opinion propranolol administration is the first option, being the second one a strong analgesic. The existence of hyperthermia should not lead us to think about an infective process as a primary cause, rather we should think about muscle heat added to hypothalamic dysfunction. These concepts should be mandatory in the knowledge of medical personnel treating patients with brain injuries, both in hospitals and in rehabilitation centers. The steps shown in Figure 1 should be followed for the detection of a possible infective process.

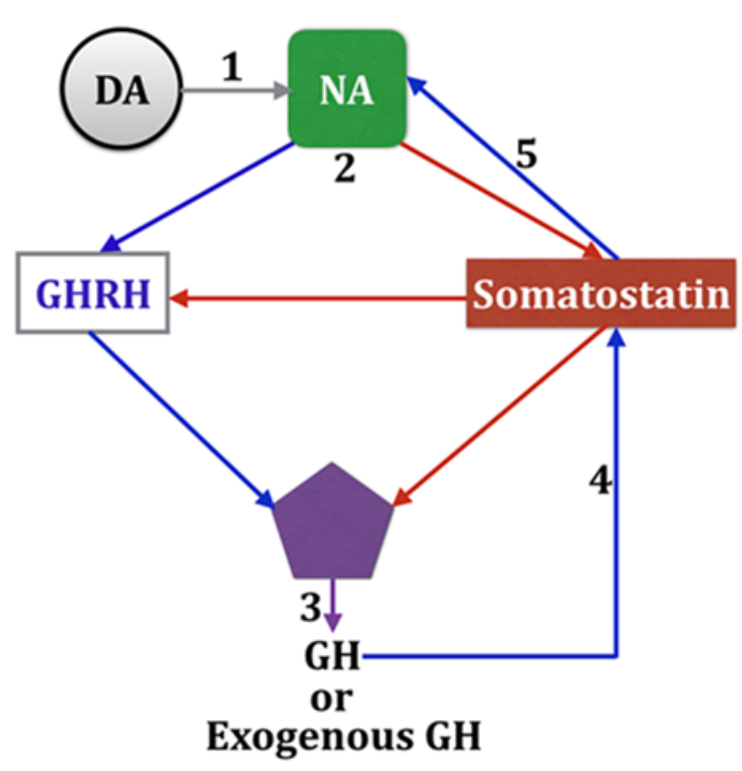

Figure I Schematic representation of the hypothalamic control of GH secretion. The pituitary secretion of $\mathrm{GH}$ is mainly regulated by the interplay between hypothalamic secretion of GHRH and somatostatin. Somatostatin negatively regulates pituitary $\mathrm{GH}$ release acting directly on somatotrophs and on the hypothalamic release of GHRH (red arrows). I, Dopamine (DA) is metabolically transformed in Noradrenaline (NA). 2, NA negatively regulates the secretion of somatostatin (red arrow) and induces the release of GHRH (blue arrow). 3, As a result of these noradrenergic effects $\mathrm{GH}$ is released to the circulation. 4, increased plasma GH levels (produced by the pituitary gland or after exogenous administration of the hormone) leads to a compensatory stimulation of the secretion of somatostatin. 5 , In turn, increased somatostatin release induces a compensatory increase in NA tone.

\section{Acknowledgements}

This study has been funded by Foundation Foltra (Teo, Spain).

\section{Conflict of interest}

Author declares that there is no conflict of interest.

\section{References}

1. Hinson HE, Sheth KN. Manifestations of the hyper adrenergic state after acquired brain injury. Curr Opin Crit Care. 2012;18(2):139-145.

2. Baguley IJ (2008) Autonomic complications following central nervous injury. Semin Neurol. 2008;28(5):716-725.

3. Meyer K (2014) Understanding paroxysmal sympathetic hyperactivity after traumatic brain injury. Surg Neurol Int. 2014;5(Suppl13):S490S492.

4. Baguley IJ, Heriseanu RE, Nott MT, et al. Dysautonomia after severe traumatic brain injury: Evidence of persisting over responsiveness to afferent stimuli. Am J Phys Med Rehabil. 2009;88(8):615-622.
5. Baguley IJ, Perkes IE, Fernandez Ortega JF, et al. Paroxysmal sympathetic hyperactivity after acquired brain injury: Consensus on conceptual definition, nomenclature, and diagnostic criteria. $J$ Neurotrauma. 2014;31(17):1515-1520.

6. Boeve BF, Wijdicks EF, Benarroch EE, et al. Paroxysmal sympathetic storms ("diencephalic seizures") after severe diffuse axonal injury. Mayo Clin Proc. 1998;73(2):148-152.

7. Rossitch E Jr, Bullard DE. The autonomic dysfunction syndrome: aetiology and treatment. Br J Neurosurg. 1988;2(4):471-478.

8. Bullard DE. Diencephalic seizures: responsiveness to bromocriptine and morphine. Ann Neurol. 1987;21(6):609-611.

9. Baguley IJ, Nicholls JL, Felmingham KL, et al. Dysautonomia after traumatic brain injury: A forgotten syndrome? J Neurol Neurosurg Psychiatry. 1999;67(1):39-43.

10. Blackman JA, Patrick PD, Buck ML, et al. Paroxysmal autonomic instability with dystonia after brain injury. Arch Neurol. 2004;61(3):321-328.

11. Perkes I, Baguley IJ, Nott MT, et al. A review of paroxysmal sympathetic hyperactivity after acquired brain injury. Ann Neurol. 2010;68(2):126-135.

12. Perkes IE, Menon DK, Nott MT, et al. Paroxysmal sympathetic hyperactivity after acquired brain injury: a review of diagnostic criteria. Brain Inj. 2011;25(10):925-932.

13. Singh DK, Singh N. Paroxysmal autonomic instability with dystonia in a child: rare manifestation of an inter peduncular tuberculoma. Pediatr Neurosurg. 2011;47(4):275-278.

14. Deepika A, Mathew MJ, Kumar SA, et al. Paroxysmal sympathetic hyperactivity in pediatric traumatic brain injury: A case series of four patients. Auton Neurosci. 2015;193:149-151.

15. Pozzi M, Conti V, Locatelli F, et al. Paroxysmal sympathetic hyperactivity in pediatric rehabilitation: clinical factors and acute pharmacological management. J Head Trauma Rehab. 2015;30(5):357-363.

16. Kern J, Bodek D, Niazi OT, et al. Refractory case of paroxysmal autonomic instability with dystonia syndrome secondary to hypoxia. Chest. 2016;149(2):e39-e40.

17. Baguley IJ. Excitatory: inhibitory ratio model (EIR Model): an integrative explanation of acute automonic overactivity syndromes. Med Hypotheses. 2008;70(1):26-35.

18. Fernandez Ortega JF, Prieto Palomino MA, Muñoz Lopez A, et al. Prognostic influence and computed tomography findings in dysautonomic crises after traumatic brain injury. J Trauma. 2006;61(5):1129-1133.

19. Lemke DM. Sympathetic storming after severe traumatic brain injury. Crit Care Nurse. 2007;27(1):30-37.

20. Baguley IJ, Nott MT, Slewa Younan S, et al. Diagnosing dysautonomia after acute traumatic brain injury: Evidence for over responsiveness to afferent stimuli. Arch Phys Med Rehabil. 2009;90(4):580-586.

21. Hinson HE, Puybasset L, Weiss N, et al. Neuroanatomical basis of paroxismal sympathetic hyperactivity: A diffusion tensor imaging analysis. Brain Inj. 2015;29(4):455-461.

22. Devesa J, Diaz MJ, Tresguerres JA, et al. Evidence that alpha 2 -adrenergic pathways play a major role in growth hormone $(\mathrm{GH})$ neuro regulation: alpha 2-adrenergic agonism counteracts the inhibitory effect of muscarinic cholinergic receptor blockade on the GH response to GHreleasing hormone, while alpha 2-adrenergic blockade diminishes the potentiating effect of increased cholinergic to neon such stimulation in normal men. J Clin Endocrinol Metab. 1991;73(2):251-256.

23. Devesa J, Lima L, Tresguerres JA. Neuroendocrine control of growth hormone secretion in humans. Trends Endocrinol Metab. 1992;3(5):175-183. 
24. Lima L, Arce V, Tresguerres JA, et al. Studies on alpha 2-adrenergic modulation of hypothalamic somatostatin secretion in rats. Life Sci. 1993;53(8):665-668.

25. Lima L, Arce V, Tresguerres JA, et al. Clonidine potentiates the growth hormone $(\mathrm{GH})$ response to $\mathrm{GH}$-releasing hormone in nor epinephrine synthesis-inhibite drats: evidence foran alpha-2-adrenergic control of hypothalamic release of somatostatin. Neuroendocrinology. 1993;57(6):1155-1160.

26. Arce V, García Barros M, Vara E, et al. Clonidine potentiates the growth hormone response to a growth hormone releasing hormone challenge in hypothalamic growth hormone releasing hormone deficient rats. Neuroendocrinology. 1995;61(5):552-558.

27. Reimunde P, Quintana A, Castañón B, et al. Effects of growth hormone $(\mathrm{GH})$ replacement and cognitive rehabilitation in patients with cognitive disorders after traumatic brain injury. Brain Inj. 2011;25(1):65-73.
28. Devesa J, Reimunde P, Devesa P, et al. Growth hormone (GH) and brain trauma. Horm Behav. 2013;63(2):331-344.

29. Arce VM, Devesa P, Devesa J. Role of growth hormone $(\mathrm{GH})$ in the treatment on neural diseases: from neuro protection to neural repair. Neurosci Res. 2013;76(4):179-186.

30. Devesa P, Agasse F, Xapelli S, et al. Growth hormone pathways signaling for cell proliferation and survival in hippocampal neural precursors from postnatal mice. BMC Neurosci. 2014;15:100.

31. Devesa P, Reimunde P, Gallego R, et al. Growth hormone (GH) treatment may cooperate with locally-produced $\mathrm{GH}$ in increasing the proliferative response of hippocampal progenitors to kainate-induced injury. Brain Inj. 2011;25(5):503-510. 